FACTA UNIVERSITATIS (NIŠ)

Ser. Math. Inform. Vol. 35, No 4 (2020), 1079-1089

https://doi.org/10.22190/FUMI2004079P

\title{
ON THE NUMERICAL RANGE OF EP MATRICES
}

\author{
Dimitrios Pappas
}

(C) 2020 by University of Niš, Serbia | Creative Commons Licence: CC BY-NC-ND

Abstract. In this work, we will study the numerical range $W(T)$ of EP matrices or operators having a canonical form $T=U(A \oplus 0) U^{*}$ in the case when $0 \notin W(A)$. As a result, we will define a kind of distance $d(W(A, T))$ between the sets $W(A)$ and $W(T)$ and investigate their connenctions, giving also upper and lower bounds for the distance $d\left(W\left(A^{-1}, T^{\dagger}\right)\right)$. Finally we will present the form of their angular numerical range $F(T)$ and its connection with $F\left(T^{\dagger}\right)$.

Keywords: Numerical Range, Angular numerical range, EP matrices, Moore-Penrose inverse.

\section{Introduction- Preliminaries and notation}

For the sake of simplicity, we will use the following notation for the unit ball of $\mathbf{C}^{n}: \quad N_{1}=\left\{x \in \mathbf{C}^{n},\|x\|=1\right\}$. All the definitions presented below can be found in $[5,7]$.

The numerical range of a square matrix $T \in \mathbf{C}^{n \times n}$ is the subset of the complex plane $\mathbf{C}$ defined as:

$$
W(T)=\left\{\langle T x, x\rangle: \quad x \in N_{1} \subset \mathbf{C}^{n}\right\} .
$$

The numerical radius $r(T)$ is defined as:

$$
r(T)=\sup \{|\lambda|, \quad \lambda \in W(T)\} .
$$

Another tool used in this work is, in the case of EP matrices $T=U(A \oplus 0) U^{*}$, the distance between the origin and the set $W(A)$, called the inner numerical radius, $\hat{r}(T)$ defined as:

$$
\hat{r}(T)=\inf \{|\lambda|, \quad \lambda \in \partial W(T)\}
$$

Received June 24, 2020; accepted September 28, 2020

2020 Mathematics Subject Classification. 15A60, 47A12 ,15A09 
Finally, the field angle $\Theta(W(T))$ is the angle formed by the two support lines of $W(T)$ coming from the origin. If $0 \in W(T)$ then $\Theta(W(T))=2 \pi$. If 0 is on the boundary of $W(T)$ and there is a unique tangent to the boundary at 0 then $\Theta(W(T))=\pi$. For more on the field angle see [7] .

When $T$ is symmetric, it holds that $r(T)=\rho(T)=\|T\|$, where $\rho(T)$ is the spectral radius of $T$. For more details, see e.g. [10].

In addition, by taking into account that when $T$ is not invertible then $N(T)$ the null space of $\mathrm{T}$ is non zero and for any vector $u \in N(T)$ we have $\langle T u, u\rangle=0$. Therefore, we conclude that $0 \in W(T)$ in the case of singular matrices.

The following result is well known and can be used for calculations and/or algorithmic purposes:

Proposition 1.1. For any given $x \in \mathbf{C}^{n}$, the numerical range $W(T)$ is equal to:

$$
W(T)=\left\{\lambda=\langle T x, x\rangle: \quad x \in N_{1} \subset \mathbf{C}^{n}\right\}=\left\{\lambda=\frac{1}{\|x\|^{2}}\langle T x, x\rangle: \quad x \in \mathbf{C}^{n}\right\}
$$

Since for any $x \in \mathbf{C}^{n}$ we have

$$
\left\{\langle T x, x\rangle=\|x\|^{2}\left\langle T\left(\frac{x}{\|x\|}\right), \frac{x}{\|x\|}\right\rangle=\|x\|^{2} \lambda, \quad \lambda \in W(T) \quad x \in \mathbf{C}^{n}\right\}
$$

The numerical range of a matrix is known to be a compact and convex subset of $\mathbf{C}$. Many interesting properties arise from this set and various properties of $\mathrm{T}$ can be deduced from $\mathrm{W}(\mathrm{T})$.

When the corresponding matrix is real, $W(T)$ is symmetric with respect to the real axis. On the other hand, $W(T) \subset \mathbf{R}$ if and only if $T$ is Hermitian, i.e., $T^{*}=T$; in this case, the endpoints of $W(T)$ coincide with the minimum and the maximum eigenvalues of T. Furthermore, $W(T)$ is a line segment in the complex plane if and only if the matrix $\mathrm{T}$ is normal and has collinear eigenvalues. We will present an example of the real case and one of of the complex case in Figure 1.1. These two examples will be used again in the sequel. Note that in both cases, the origin does not belong to $W(T)$.

Another tool used in this work is the generalized inverse (Moore-Penrose) of a singular square matrix. (Since the Numerical Range is defined only for square matrices.) In the case when $T$ is a complex $m \times n$ matrix of rank $r$, Penrose showed that there is a unique matrix satisfying the four Penrose equations, called the pseudo-inverse of $T$, denoted by $T^{\dagger}$ such that

$$
T T^{\dagger}=\left(T T^{\dagger}\right)^{*}, \quad T^{\dagger} T=\left(T^{\dagger} T\right)^{*}, \quad T T^{\dagger} T=T, \quad T^{\dagger} T T^{\dagger}=T^{\dagger},
$$

where $T^{*}$ denotes the conjugate transpose of $T$.

It is easy to see that $T T^{\dagger}$ is the orthogonal projection of $\mathbf{C}^{n}$ onto the range $\mathcal{R}(T)$, of $T$, denoted by $P_{T}$, and that $T^{\dagger} T$ is the orthogonal projection of $\mathbf{C}^{m}$ onto $\mathcal{R}\left(T^{*}\right)$, 

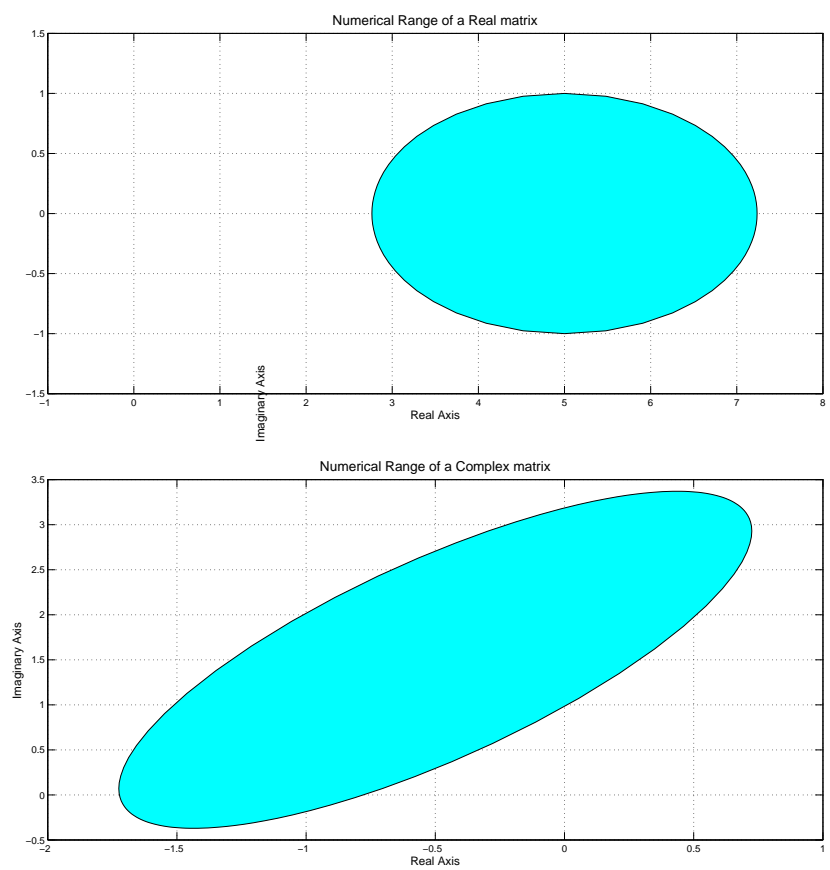

FIG. 1.1: The numerical Range of (a) a real and (b) a complex matrix.

denoted by $P_{T^{*}}$. It is also well known that it holds $\mathcal{R}\left(T^{\dagger}\right)=\mathcal{R}\left(T^{*}\right)$. Standard reference books on generalized inverses are $[1,2,4]$.

The matrix $T$ is called EP matrix if $T T^{\dagger}=T^{\dagger} T$. The set of EP matrices of rank $r$ are usually denoted by $\mathrm{EP}_{r}$. We take advantage of the fact that EP matrices have a simple canonical form according to the decomposition $\mathbf{C}^{m}=\mathcal{R}(T) \oplus \mathcal{N}(T)$. Indeed, an $\mathrm{EP}_{r}$ matrix $T$ has the simple block matrix form

$$
T=U\left[\begin{array}{cc}
A & 0 \\
0 & 0
\end{array}\right] U^{*}=U(A \oplus 0) U^{*},
$$

where the matrix $A: R(T) \rightarrow R(T)$ is invertible with $\operatorname{rank}(A)=r$ and $U$ is unitary. So, $T$ can aslo be seen as a dilation of the matrix $A$.

The generalized inverse $T^{\dagger}$ of the matrix $T$ defined in (1.2) has the form

$$
T^{\dagger}=U\left[\begin{array}{cc}
A^{-1} & 0 \\
0 & 0
\end{array}\right] U^{*}=U\left(A^{-1} \oplus 0\right) U^{*} .
$$

This decompositon is called the $U R U^{*}$ decomposition of a matrix, and is a special case of the $U R V$ decomposition.

According to another characterization, a square complex matrix $T$ is said to be EP if $T$ and its conjugate transpose $T^{*}$ have the same range. EP operators matrices 
constitute a wide class, which includes the self adjoint, the normal and the invertible matrices. For more details about on EP matrices we refer to [2, 3, 11]. Various characterizations of EP matrices were also collected in [12].

All the previous results are also valid for bounded EP operators on Hilbert space with the appropriate modifications.

Throughout this paper, $\mathcal{H}$ will denote a separable Hilbert space of infinite dimension and the set of all bounded operators acting on $\mathcal{H}$ is denoted by $B(\mathcal{H})$. When the space is finite dimensional, $\mathcal{H}$ will be replaced by $\mathbf{C}^{n}$.

\section{The Numerical Range of EP matrices}

An important role, studying numerical ranges, plays whether or not zero belongs to the numerical range. Necessary and sufficient conditions such that the origin belongs to the numerical range of a complex matrix may be found e.g. in [9]. In this work, we will examine the special case of EP matrices, $T=U(A \oplus 0) U^{*}$ such that $0 \notin W(A)$.

For any matrix $A \in \mathbf{C}^{n \times n}$, and any principal submatrix $A_{1}$ of $\mathrm{A}, W\left(A_{1}\right) \subseteq W(A)$. In the case of an EP operator, we have the following:

Theorem 2.1. Let $T$ be an EP operator with the simple canonical form according to the decomposition $\mathcal{H}=\mathcal{R}(T) \oplus \mathcal{N}(T)$, having the form : $T=U(A \oplus 0) U^{*}$.

Then $W(T)=c o(W(A) \cup(0))$.

Proof. It is obvious that $W(T) \supseteq c o(W(A) \cup(0))$. For the contrary, since the space can be decomposed as $\mathcal{H}=\mathcal{R}(T) \oplus \mathcal{N}(T)$, then for every $x \in \mathcal{H}, x=x_{1}+x_{2}$.

If $\lambda \in W(T), \lambda \neq 0$ then $\lambda=\langle T x, x\rangle=\left\langle A x_{1}, x_{1}\right\rangle$ for some $x,\|x\|=1$, therefore $\lambda=\left\langle A x_{1}, x_{1}\right\rangle=\lambda^{\prime}\left\|x_{1}\right\|^{2}$ with $\left\|x_{1}\right\| \leq 1$ and $\lambda^{\prime} \in W(A)$. This is equal to

$$
\lambda=\lambda^{\prime}\left\|x_{1}\right\|^{2}+\left(1-\left\|x_{1}\right\|^{2}\right) 0,
$$

so $\lambda \in \operatorname{co}(W(A) \cup(0))$. Obviously, when $0 \in W(A) \Rightarrow W(A)=W(T)$.

The above result shows clearly the fact that when a number $\lambda$ is a corner of $W(A)$ then $\lambda$ is an eigenvalue of $A$ (see [8]).

Example 2.1. We give two particular examples of the above proposition in the next two figures.

1. Let

$$
A=\left[\begin{array}{ll}
4 & 1 \\
3 & 6
\end{array}\right], \quad T=\left[\begin{array}{lll}
4 & 1 & 0 \\
3 & 6 & 0 \\
0 & 0 & 0
\end{array}\right]
$$

A is the real matrix whose Numerical range was shown in Figure 1.1(a)

We have seen that $0 \notin W(A)$. The numerical range of $\mathrm{A}$ is presented in blue in Figure 2.1. The numerical range of $T=U(A \oplus 0) U^{*}$ is presented in green. $W(A)$ is an ellipse, with foci the eigenvalues of $\mathrm{A}, \lambda_{1}=3, \lambda_{2}=7$ shown in red. We can see that $W(T)$ is the convex hull of $W(A) \cup(0)$ and that the origin is a corner point of $W(T)$. 


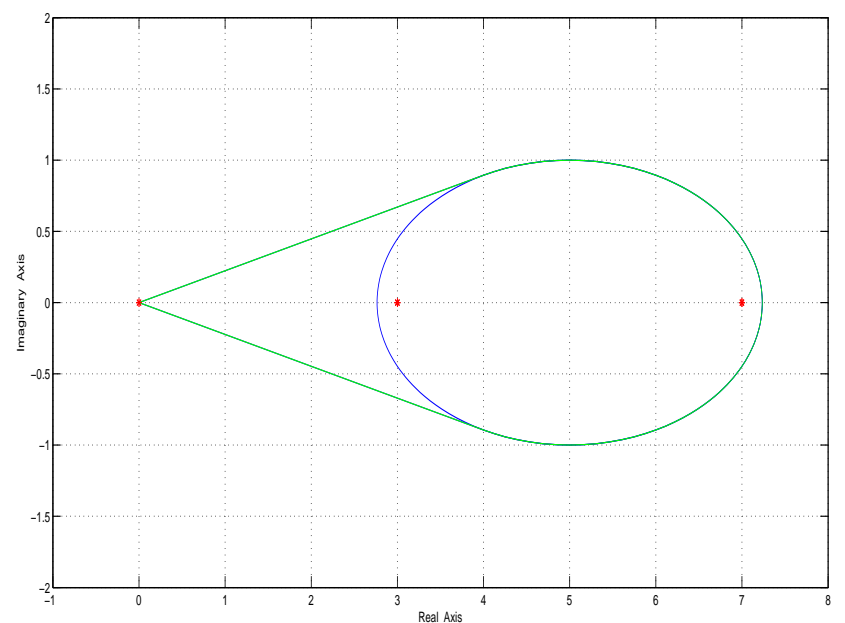

FIG. 2.1: Numerical Range of $W(T)=c o(W(A) \cup(0))$ in green, $W(A)$ in blue.

2. Let

$$
B=\left[\begin{array}{cc}
-1 & i \\
2 & 3 i
\end{array}\right], \quad S=\left[\begin{array}{ccc}
-1 & i & 0 \\
2 & 3 i & 0 \\
0 & 0 & 0
\end{array}\right]
$$

$\mathrm{B}$ is the complex matrix whose Numerical range was shown in Figure 1.1(b). As we have seen in Figure 1.1(b), $0 \notin W(B)$ and $W(B)$ is an ellipse, with foci the eigenvalues of $\mathrm{B}$, $\lambda_{1}=1.508-0.236 i, \lambda_{2}=0.508+3.24 i$. The numerical range $W(S)$ of $S=U(B \oplus 0) U^{*}$ is presented in green in Figure 2.2 and the eigenvalues are shown in red. We can see again that the origin is a corner point of $W(S)$ and that $W(S)$ is the convex hull of $W(B) \cup 0$.

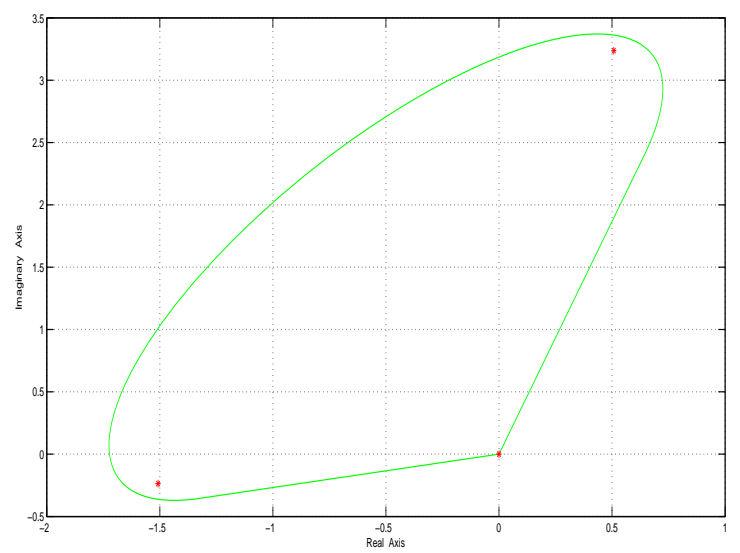

FIG. 2.2: Numerical Range of $W(S)=c o(W(B) \cup(0))$ in green. 


\section{The distance between $W(T), W(A)$}

As we can see from the previous results for EP operators the numerical range $W(T)$ is an extension of the numerical range $W(A)$. To what extend? To have a measure of this we define a kind of distance between the sets $W(T)$ and $W(A)$. In fact, we use the distance $d(W(A, T))$ of the origin to $W(A)$. Whenever $0 \in W(A)$ then the two sets coincide, so the distance is equal to zero. When $0 \notin W(A)$ then $d(W(A, T))=\hat{r}(A)$, the inner numerical radius of $A$, which is defined as $\hat{r}(A)=\min |z|, z \in W(A)$. We give the following definition:

Definition 3.1. Let $T \in B(\mathcal{H})$ be an EP operator having a canonical form $T=$ $U(A \oplus 0) U^{*}$. Then the distance between the numerical ranges $W(A), W(T)$ is defined as:

$$
d(W(A, T))=\left\{\begin{array}{cc}
0, & 0 \in W(A) \\
\hat{r}(A), & 0 \notin W(A)
\end{array}\right.
$$

As we can see, this type of distance may be seen as a special case of the Hausdorff distance between subsets of a metric space.

Using the matrices presented in Example 2.1, we have that

$$
d(W(A, T))=2.7639, \quad d(W(B, S))=0.5711
$$

The calculation of the above values was made using a modified Matlab code presented in [10].

A natural question then would be: What about the distance $d\left(W\left(A^{-1}, T^{\dagger}\right)\right)$, and its relation with $d(W(A, T))$ ? Equivalently, what is the relation between $\hat{r}(A), \hat{r}\left(A^{-1}\right)$ when $0 \notin W(A)$ ?

We obviously have that $T^{\dagger}=U\left[\begin{array}{cc}A^{-1} & 0 \\ 0 & 0\end{array}\right] U^{*}$.

It also holds that $W\left(T^{\dagger}\right)=c o\left(W\left(A^{-1}\right) \cup(0)\right)$. Since in general there is no connection between $W(A)$ and $W\left(A^{-1}\right)$, neither between the numerical radius of these matrices, the only relation we can have is an inequality connecting $d\left(W\left(A^{-1}, T^{\dagger}\right)\right)$, and $d(W(A, T))$.

An answer can be given using a result from [6]:

Proposition 3.1. Let a nonsingular square matrix $A, \hat{r}(A)$ denote its inner numerical radius and $r(A)$ its numerical radius. Then it holds that:

$$
\frac{\hat{r}(A)}{\|A\|^{2}} \leq \hat{r}\left(A^{-1}\right) \leq \min \left\{\frac{\hat{r}(A)}{\sigma_{\min }^{2}(A)}, \frac{r(A)}{\|A\|^{2}}\right\}
$$

Based on the above result, we conclude the following Proposition:

Proposition 3.2. Let an EP matrix $T$ with the canonical form $T=U(A \oplus 0) U^{*}$. If $0 \notin W(A)$ then we have

$$
\frac{d(W(A, T))}{\|A\|^{2}} \leq d\left(W\left(A^{-1}, T^{\dagger}\right)\right) \leq \min \left\{\frac{d(W(A, T))}{\sigma_{\min }^{2}(A)}, \frac{r(A)}{\|A\|^{2}}\right\}
$$


Example 3.1. Using the matrices from Example 2.1:

$$
A=\left[\begin{array}{ll}
4 & 1 \\
3 & 6
\end{array}\right], \quad T=\left[\begin{array}{lll}
4 & 1 & 0 \\
3 & 6 & 0 \\
0 & 0 & 0
\end{array}\right] \quad T^{\dagger}=\left[\begin{array}{ccc}
0.2857 & -0.0476 & 0 \\
-0.1429 & 0.1905 & 0 \\
0 & 0 & 0
\end{array}\right]
$$

we have that :

$r(A)=7.2361$ and $\hat{r}(A)=2.7639$, while $\|A\|=7.3351, \quad \sigma_{\min }(A)=3$.

So, replacing in the above inequality we can see that

$$
\begin{gathered}
\frac{2.7639}{7.3351^{2}} \leq d\left(W\left(A^{-1}, T^{\dagger}\right)\right) \leq \min \left\{\frac{2.7639}{9}, \frac{7.2361}{7.3351^{2}}\right\} \Leftrightarrow \\
0.0514 \leq d\left(W\left(A^{-1}, T^{\dagger}\right)\right) \leq 0.1345
\end{gathered}
$$

Using Matlab we get that $d\left(W\left(A^{-1}, T^{\dagger}\right)\right)=\hat{r}\left(A^{-1}\right)=0.1316$ which satisfies the above inequality.

Another question on the relation between $W(A), W(T)$ is how much the boundary $\partial(A)$ has been changed to give $\partial(T)$. The distance $d(W(A, T))$ can give us a measure of course, but we can also perform a statistical analysis of the boundaries of numerical ranges as another index of the change performed. We expect the variance of $\partial(T)$ to increase more with respect to $\partial(A)$, as the distance $d(W(A, T))$ increases.

Example 3.2. In this example we will use the matrices B, S from Example 2.1 and the following matrices $\mathrm{C}, \mathrm{R}$ :

$$
C=\left[\begin{array}{cccc}
4 & 0 & 0 & -1 \\
-1 & 4 & 0 & 0 \\
0 & -1 & 4 & 0 \\
0 & 0 & -1 & 4
\end{array}\right] \quad R=\left[\begin{array}{ccccc}
4 & 0 & 0 & -1 & 0 \\
-1 & 4 & 0 & 0 & 0 \\
0 & -1 & 4 & 0 & 0 \\
0 & 0 & -1 & 4 & 0 \\
0 & 0 & 0 & 0 & 0
\end{array}\right]
$$

We have that $d(W(C, R))=\hat{r}(C)=3$ and we can calculate the variance of the boundaries of $\mathrm{C}$ and $\mathrm{R}$ :

$$
\operatorname{Var}(\partial(C))=0.520, \quad \operatorname{Var}(\partial(R))=4.946 \text {. }
$$

On the other hand, using the matrices B, S from Example 2.1 with $S=U(B \oplus 0) U^{*}$, having a much lower distance of the Numerical Ranges, $d(W(B, S))=0.5711$ we can see that:

$$
\operatorname{Var}(\partial(B))=0.949, \quad \operatorname{Var}(\partial(S))=0.90958 .
$$

As another possible index of change, we can also calculate the field angles $\Theta(W(R))=0.49$ radians and $\Theta(W(S))=2.074$ radians, using the matlab code drawing the Numerical range found in $[10]$.

From the above results we cannot have a clear picture of the relation between $d(W(A, T)$ and the variance of the boundaries or the field angle. So, we will return to this question in the last section (discussion) of this paper. 


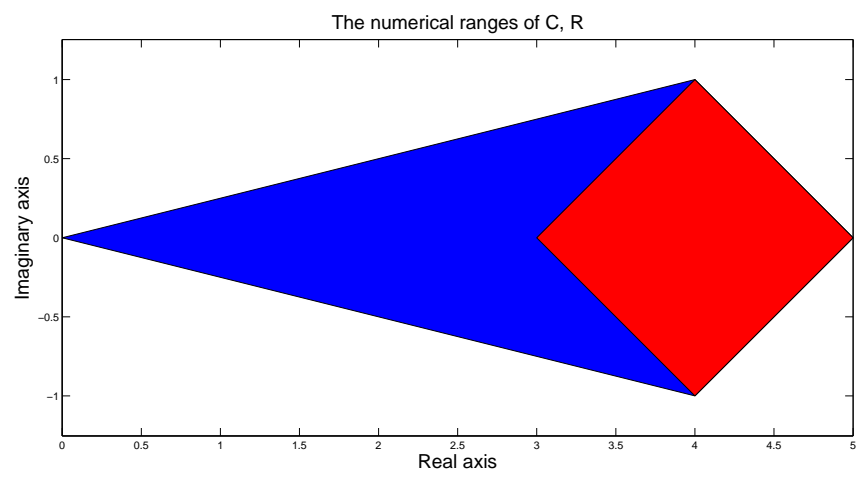

FIG. 3.1: Numerical Range of $W(R)=c o(W(C) \cup(0))$ in blue and red, $W(C)$ in red.

\section{The angular numerical range of EP operators and matrices}

As we have seen, the origin is a sharp point of $W(T)$ in the above examples. This property leads us to the notion of the Angular Numerical Range, a cone of the complex plane and we will discuss it in this section. The angular opening of the smallest angular sector including $W(T)$ is the field angle $\Theta(W(T))$ and is also the angular opening of the Angular Field of values $F(T)$ :

Definition 4.1. The Angular Field of Values (or Angular Numerical range) of an operator T, denoted by $F(T)$ is the subset of the complex plane $\mathbf{C}$ defined as:

$$
F(T)=\left\{\langle T x, x\rangle: \quad x \in \mathbf{C}^{n}, x \neq 0\right\} .
$$

It is clear that $F(T)$ is a cone or a sector in $\mathbf{C}$ having its top at the origin. It is known that $F(T)$ is spanned by $W(T)$ and that $\Theta(W(T))=\Theta(F(T))$.

In the case of nonsingular operators or matrices, we know that there is no connection between the numerical range $W(T)$ and of $W\left(T^{-1}\right)$. But when it comes to the angular numerical range, $F(T)$, then $F(T)=\overline{F\left(T^{-1}\right)}$, where $\overline{F\left(T^{-1}\right)}$ denotes the conjugate set. (See [7] page 66). It is clear from this result that for real matrices we have $F\left(T^{-1}\right)=F(T)$.

But, what happens if we replace $T^{-1}$ with $T^{\dagger}$ in the case of a singular operator or matrix?

In this case we can notice the following: When $T$ is singular, then 0 always belongs to $W(T)$ ( take a vector $x$ in the kernel of $T$ ). In the general case when 0 is in the interior of the numerical range, then $F(T)$ is the entire complex plane, and then $F(T)=F\left(T^{\dagger}\right)=\mathbf{C}$.

We will examine the non trivial case, the class of EP operators, because then, 0 is an angular point of the numerical range. In this case we have the following theorem:

Theorem 4.1. When $T$ is a singular EP operator, $T=U(A \oplus 0) U^{*}, \quad 0 \notin W(A)$. Then $F\left(T^{\dagger}\right)=\overline{F(T)}$. In the case of real matrices, it holds that $F\left(T^{\dagger}\right)=F(T)$. 
Proof. By taking the canonical form of $T$, there exists a unitary operator $U$ and an invertible operator (or an $r \times r$ matrix) $A$, such that

$$
T=U\left[\begin{array}{cc}
A & 0 \\
0 & 0
\end{array}\right] U^{*}
$$

Using this factorization, and using the fact that $F(T)$ is preserving congruence (See [7], page 13) we can see that $F(T)=F(A)$. Indeed, from the definition of $F(T)$, we have that ( since $\mathcal{H}=\mathcal{R}(T) \oplus \mathcal{N}(T)$ ):

$$
F(T)=F(A \oplus 0)=\left[\begin{array}{ll}
x^{*} & y^{*}
\end{array}\right]\left[\begin{array}{cc}
A & 0 \\
0 & 0
\end{array}\right]\left[\begin{array}{l}
x \\
y
\end{array}\right]=x^{*} A x=F(A)
$$

Now, using the fact that

$$
T^{\dagger}=U\left[\begin{array}{cc}
A^{-1} & 0 \\
0 & 0
\end{array}\right] U^{*}
$$

and that $F(T)=\overline{F\left(T^{-1}\right)}$, we have that $F\left(T^{\dagger}\right)=F\left(A^{-1}\right)=\overline{F(A)}=\overline{F(T)}$.

In Figure 4.1 that follows we can see that the Field Angles and the Angular Numerical Ranges (the blue and green cones anchored in the origin) coincide for the matrices $R, R^{\dagger}$ used in Example 3.2, and also presented in Figure 3.1. As we can see, the angular opening of the sectors $F(R), F\left(R^{\dagger}\right)$ is the same:

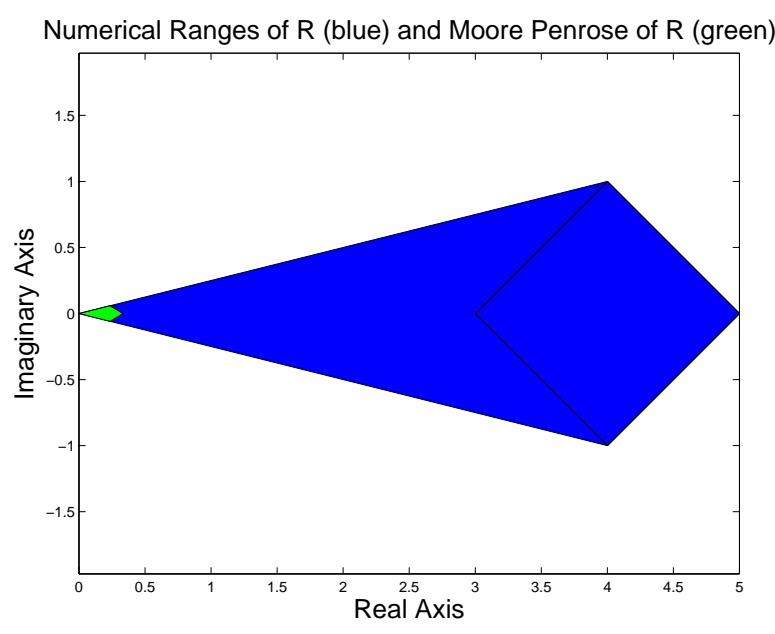

Fig. 4.1: Numerical Ranges and Angular Numerical Ranges of the real matrices $R, R^{\dagger}$ in blue and green respectively. We can see that $F(R)=F\left(R^{\dagger}\right)$. 
Table 5.1: Different values of $d(W(A, T)), \Theta(W(A)), \operatorname{Var}(A)$ where $T=U(A \oplus 0) U^{*}$

\begin{tabular}{llll}
\hline Matrix & $d(W(A, T))$ & $\Theta(W(T))$ & $\operatorname{Var}(\partial(T))$ \\
\hline $\mathrm{P}$ & 15.52 & 1.6562 & 3.4704 \\
$\mathrm{R}$ & 3 & 0.49 & 4.95 \\
$\mathrm{~T}$ & 2.764 & 0.44 & 10.81 \\
$\mathrm{~S}$ & 0.571 & 2.075 & 0.909 \\
\hline
\end{tabular}

\section{Discussion- Conclusion}

In this work, we presented a detailed analysis of the numerical range of EP operators and matrices and its connection with their canonical form. We defined the distance $d(W(T, A))$ when $T$ can be decomposed as $T=U(A \oplus 0) U^{*}$.

From all the above discussion, we can say that for EP operators and matrices, the Numerical range $W(T)$ can be seen as an pertrubation of $W(A)$ when $0 \notin W(A)$. But, to what extend is this pertrubation? One can think by geometric intuition that in general, when $d(W(A, T))$ gets larger then the field angle $\Theta(W(T))$ gets smaller, while the variance of the numerical boundary gets larger also.

Investigating this assumption, we used some numerical examples of matrices with different sizes and various values of the distance $d(W(A, T))$ defined in this section. In the following table we can see the corresponding values for the matrices presented in the examples of this paper. The matrix $\mathrm{P}$ is a random $7 \times 7 \mathrm{EP}$ matrix of the form $P=U\left(P_{1} \oplus 0\right) U^{*}, 0 \notin W\left(P_{1}\right)$.

From the results presented in the above table we can say that this question needs a deeper investigation, since we can see that the general trend that we expected is satisfied but not always. More factors have to be taken into account in order to have a more clear picture of the connection between $d(W(A, T)), \Theta(W(T))$. This gives us a motivation for the extention of this work in the future.

The other topic that was presented in this work was the Angular Numerical range $F(T)$. The connection of $F(T)$ and $F\left(T^{-1}\right)$ has already been investigated (see [7]), and in this work we extended this result for $F\left(T^{\dagger}\right)$ for the class of EP operators and matrices.

\section{REF ER E N C E S}

1. A. Ben-Israel, T. N. E. Grenville: Generalized Inverses: Theory and Applications, Springer- Verlag, Berlin, (2002)

2. S. L. Campbell, C. D. Meyer: Generalized Inverses of Linear Transformations, Dover Publications Inc., New York, (1991)

3. Drivaliaris D., Karanasios S., Pappas D.: Factorizations of EP operators, Linear Algebra and Applications, 429, 1555-1567 (2008) 
4. C. W. Groetsch, Generalized inverses of linear operators, Marcel Dekker Inc. New York (1977)

5. K.E. Gustafson and D.K.M. Rao, Numerical Range, Springer, New York (1997)

6. Hochstenbach M. E., Singer, D. A., Zachlin, P. F.: Numerical approximation of the field of values of the inverse of a large matrix. (CASA-report; Vol. 1308). Eindhoven: Technische Universiteit Eindhoven. (2013)

7. R. Horn, C. Johnson: Topics in Matrix Analysis, Cambridge Univ. Press (1991)

8. H. Langer, A. Markus, and C. Tretter: Corners of numerical ranges, Operator Theory: Advances and Applications, 124, 383-400 (2001)

9. L. Knockaert: Necessary and sufficient conditions for the origin to belong to the numerical range of a matrix WSEAS Trans. Math., 5, 1350-1352 (2006)

10. P. Psarrakos, M. Tsatsomeros: Numerical Range (in) a matrix nutshell, Mathematics Notes (V. 155) Department of Mathematics, Washington State University, (2002)

11. H. Schwerdtfeger, Introduction to Linear Algebra and the Theory of Matrices, P. Noordhoff, Groningen, (1950)

12. Y. Tian: Characterizations of EP matrices and weighted EP matrices, Linear Algebra and its Applications 434(5), 1295-1318 (2011)

\author{
Dimitrios Pappas \\ Athens University of Economics and Business \\ Department of Statistics, \\ and Stochastic Modeling and Applications Laboratory \\ Athens, Greece \\ 76 Patission Str \\ 10434 Athens, Greece \\ dpappas@aueb.gr, pappdimitris@gmail.com
}

